\title{
Kidins220/ARMS is an essential modulator of cardiovascular and nervous system development
}

\author{
F Cesca ${ }^{*, 1,6}$, A Yabe ${ }^{2,6}$, B Spencer-Dene ${ }^{3}$, A Arrigoni ${ }^{1}$, M Al-Qatari ${ }^{4}$, D Henderson ${ }^{5}$, H Phillips ${ }^{5}$, M Koltzenburg ${ }^{4}$, F Benfenati ${ }^{1,7}$ \\ and G Schiavo $0^{2,7}$
}

The growth factor family of neurotrophins has major roles both inside and outside the nervous system. Here, we report a detailed histological analysis of key phenotypes generated by the ablation of the Kinase $D$ interacting substrate of $220 \mathrm{kDa} / A n k y$ rin repeat-rich membrane spanning (Kidins220/ARMS) protein, a membrane-anchored scaffold for the neurotrophin receptors Trk and $\mathrm{p} 75^{\mathrm{NTR}}$. Kidins220 is important for heart development, as shown by the severe defects in the outflow tract and ventricle wall formation displayed by the Kidins220 mutant mice. Kidins220 is also important for peripheral nervous system development, as the loss of Kidins220 in vivo caused extensive apoptosis of DRGs and other sensory ganglia. Moreover, the neuronal-specific deletion of this protein leads to early postnatal death, showing that Kidins220 also has a critical function in the postnatal brain. Cell Death and Disease (2011) 2, e226; doi:10.1038/cddis.2011.108; published online 3 November 2011

Subject Category: Neuroscience

Companion paper of: doi:10.1038/cdd.2011.141

The development of the peripheral (PNS) and central (CNS) nervous systems is the result of a carefully orchestrated cooperation between several trophic factors. During development, distinct neuronal populations rely on a specific combination of growth factors, which activate unique intracellular responses depending on the availability of adaptors and scaffolding proteins in the target cells. The data described in this work, together with the findings presented in the accompanying paper, ${ }^{1}$ demonstrate that Kidins220 is a multifaceted protein performing pleiotropic functions in several organs both during embryonic development and postnatal life. Neurotrophins (NTs) were amongst the first trophic cues to be identified due to their crucial role in the survival of peripheral neurons. ${ }^{2}$ After decades of intense investigation, NTs, which include nerve growth factor (NGF), brain-derived neurotrophic factor (BDNF), neurotrophin-3 (NT3) and -4/5 (NT4/5), are now known to govern almost every aspect of neuronal physiology, as they modulate neuronal survival, migration, differentiation and synaptic plasticity. $^{3}$ The role of NTs as survival factors is particularly evident during PNS development. Analysis of NT- and Trk-deficient mouse lines has, in fact, revealed a heavy loss in almost all sensory ganglia, including trigeminal, nodose-petrosal, vestibular, cochlear and dorsal root ganglia. ${ }^{3}$ In general, most sensory neurons express one Trk receptor during development, but in some cases a switch in NT dependence at different developmental stages has been reported, as shown for trigeminal $^{4}$ and DRG neurons. ${ }^{5}$

The physiological functions of NTs, however, extend well beyond the nervous system. Among the extra-neuronal functions of NTs, which include the development and maintenance of several organs, of particular interest is their role during cardiovascular development. $\mathrm{BDNF}^{-1-}$ mice display atrial septal defects, intramyocardial haemorrhage and perivascular oedema at intramyocardial arterioles, resulting in defects in contractility. ${ }^{6}$ TrkB $^{-1-}$ mice display a reduction in the number of developing coronary vessels, particularly in the subepicardial region, as well as apoptosis in the same area. ${ }^{7}$ Upon ablation of NT3, the heart becomes enlarged and globular, with dilated atria, thinning of atrial wall, and atrial septal defects. Alterations are observed in the outflow tracts, with premature closure of ductus arteriosus, overriding aorta, and valvular defects. ${ }^{8}$ TrkC $^{-1-}$ mice also display enlargement of the heart with valvular, atrial and ventricular septal defects, ${ }^{9}$ which are likely caused by cardiac neural crest defects. ${ }^{10}$ Cardiac defects have not been reported in $\mathrm{p} 75^{\mathrm{NTR}-/-}$ mice, but these mutant animals develop overt defects in large blood vessels, such as the dorsal aorta, which includes ruptures and haemorrhaging. ${ }^{11}$

Cross-talk and modulation of different growth factor receptors on the same target cell is crucial for neuronal

\footnotetext{
${ }^{1}$ Department of Neuroscience and Brain Technologies, The Italian Institute of Technology, Genoa, Italy; ${ }^{2}$ Molecular Neuropathology Laboratory, Cancer Research UK London Research Institute, London, UK; ${ }^{3}$ Experimental Pathology Laboratory, Cancer Research UK London Research Institute, London, UK; ${ }^{4}$ Institute of Child Health, University College of London, London, UK and ${ }^{5}$ Institute of Genetic Medicine, Newcastle University, UK

${ }^{*}$ Corresponding author: F Cesca, Department of Neuroscience and Brain Technologies; The Italian Institute of Technology, Via Morego, 30 - 16163 Genova, Italy. Tel: + 390107178 1788; Fax: + 390107178 1230; E-mail: fabrizia.cesca@iit.it

${ }^{6}$ These authors contributed equally to this work.

${ }^{7}$ These authors contributed equally to this work.

Keywords: Kidins220/ARMS; neuronal death; heart development; sensory ganglia

Abbreviations: BDNF, brain-derived neurotrophic factor; CGRP, calcitonin gene-related peptide; CNS, central nervous system; DIV, days in vitro; DRG, dorsal root ganglia; Kidins220/ARMS, kinase D-interacting substrate of $220 \mathrm{kDa} /$ ankyrin repeat-rich membrane spanning; MAPK/Erk, mitogen activated protein kinase/extracellular signal activated kinase; NFH, neurofilament H; NGF, nerve growth factor; NT, neurotrophin; p75 ${ }^{\mathrm{NTR}}$, p75 neurotrophin receptor; per, peripherin; PNS, peripheral nervous system; PV, parvalbumin; SVZ, subventricular zone; Trks, tropomyosin-related kinase receptors

Received 14.9.11; accepted 21.9.11; Edited by G Melino
} 
survival and development. For example, the NT receptor p75 ${ }^{\text {NTR }}$ can interact with Trk, sortilin and Nogo receptors. ${ }^{12}$ Similarly, the cross-talk of GDNF- $\alpha$ receptor, RET tyrosine kinase and protocadherins modulates neuronal survival, ${ }^{13}$ while EphB and NMDA receptors cooperate in the formation of excitatory synapses. ${ }^{14}$ Intracellular scaffolding proteins have a pivotal role in this coordination, by keeping different receptors in close proximity, thus allowing cross-talk upon stimulation, together with preassembled complexes for downstream signaling. For example, large presynaptic proteins such as bassoon and piccolo, or PSD95 in the postsynaptic compartment, are involved in multiple protein-protein interactions and coordinate important processes, such as cytoskeletal dynamics, synaptic vesicle fusion, trafficking of AMPA and NMDA receptors and many others. ${ }^{15-17}$

Kidins220/ARMS (Kinase D interacting substrate of $220 \mathrm{kDa} /$ Ankyrin repeat-rich membrane spanning) is a multifunctional scaffold protein that is able to interact with a number of transmembrane receptors, such as Trks and $p 75^{\mathrm{NTR}},{ }^{18-20}$ Eph, ${ }^{21}$ AMPAR $^{22}$ NMDAR $^{23}$ and VEGFR. ${ }^{1}$ As such, its deletion in vivo is expected to cause multiple developmental defects. In the accompanying paper, we report the generation and functional characterisation of a full Kidins220 knockout mouse strain. ${ }^{1}$ As expected, constitutive Kidins220 ablation resulted in widespread neuronal death in both CNS and PNS. However, we also unveiled an unexpected role of Kidins220 in brain vascular development, and in heart formation. ${ }^{1}$ Here, we sought to characterise in more detail some of the phenotypes displayed by Kidins220 mutant animals, focusing on cardiovascular and sensory neuron development. These results, together with evidence in the literature, put Kidins220 at the centre of a complex signaling network, mediating the activation of specific pathways in a cell- and tissue-specific manner.

\section{Results}

Kidins220 expression levels in wild-type and heterozygous animals. To evaluate Kidins220 expression levels in our mutant mice, we took advantage of antibodies raised against the amino- ${ }^{24}$ and carboxy- ${ }^{25}$ terminal portions of the protein. As shown in Figure 1a, we could not detect any specific signal in Kidins $220^{-l-}$ samples, thus excluding the possibility of expression of truncated non-functional Kidins220 fragments in the knockout tissue. Recently, another mouse line lacking Kidins220 was described, where heterozygous mice displayed a $30-40 \%$ reduction in the protein levels of Kidins $220 .^{26}$ To evaluate the amount of protein in our Kidins $220^{+/-}$animals, we dissected brains from wild-type and heterozygous littermates at various developmental stages. Kidins220 levels in different brain regions were evaluated by western blot and densitometric analysis. We found a comparable amount of protein in all the brain regions analysed up to 1 year of age (Figures $1 b$ and $c$ ). These results suggest that our heterozygous animals are equivalent to wild-type controls in terms of Kidins220 expression. Therefore, we restricted our studies to wild-type and Kidins $220^{-1-}$ embryos.

Kidins220 ${ }^{-l-}$ embryos show defects in cardiac development. Kidins $220^{-/-}$embryos display striking
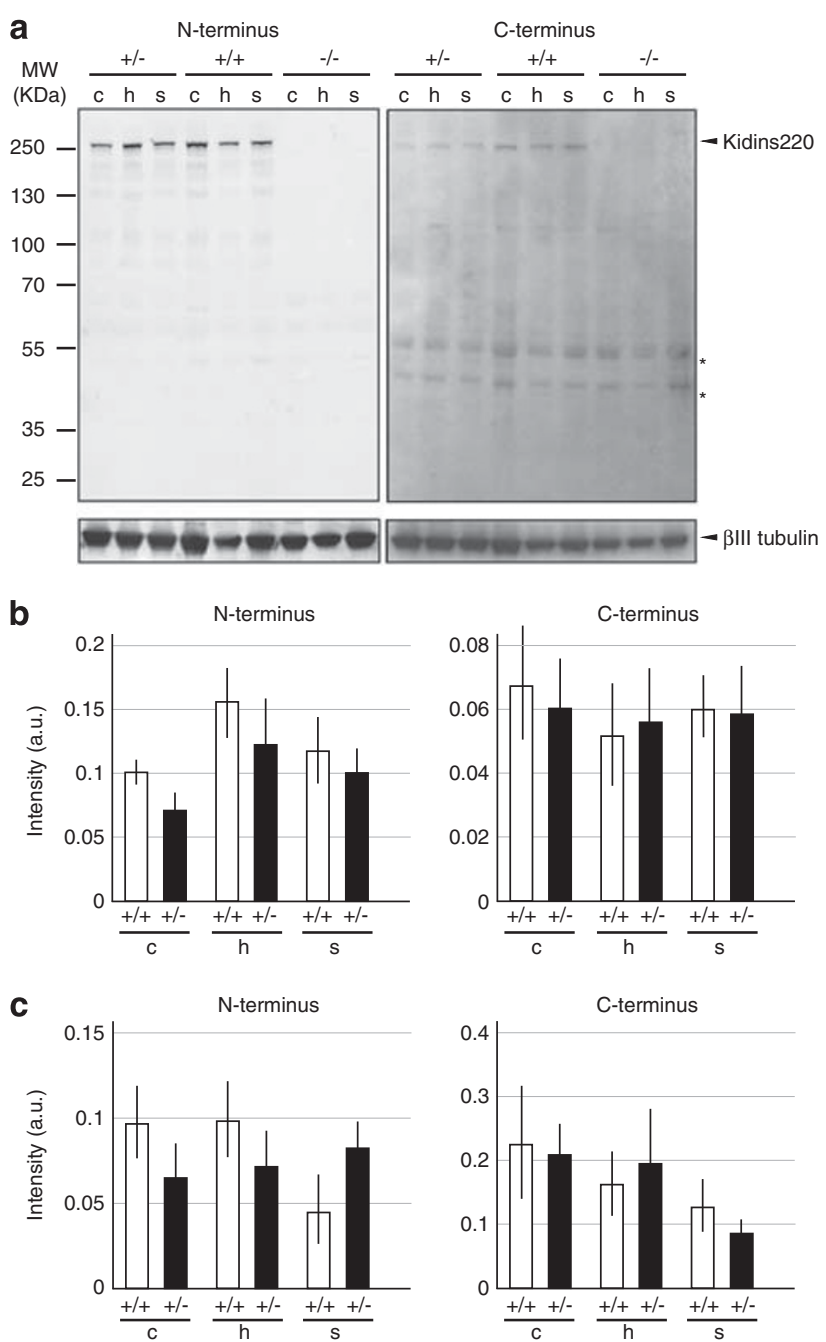

Figure 1 Kidins220 expression levels in wild-type and Kidins $220^{+1-}$ animals. (a) Cortex (c), hippocampus (h) and striatum (s) were dissected from wild-type $(+/+)$, Kidins $220^{+/-}$or Kidins $220^{-1-}$ littermates at various ages, lysed and run on a SDS-PAGE. Western blots were then incubated with antibodies against the $\mathrm{N}$-terminus (left) or the C-terminus (right) of Kidins220. (a) Representative blots from E18.5 embryos. The C-terminal antibody detected non-specific low molecular weight bands (asterisks). Anti- $\beta$ III tubulin antibodies were used to show equal loading. (b and c) Quantification of Kidins220 expression levels in wild-type and heterozygous E18.5 embryos (b) and 1 year old adult animals (c). Immunoreactive bands were quantified by using the ImageQuant TL software. The amount of Kidins220 was normalized to the amount of $\beta I I I$ tubulin, and shown as means \pm S.E.M. Comparable amounts of Kidins220 were present in wild-type and Kidins $220^{+1-}$ samples in all the brain regions and at all ages analyzed ( $P>0.05$, Student's unpaired $t$-test; $n=4$ embryos for each genotype)

developmental heart defects. As shown in Figure 2A, the morphology of the Kidins $220^{-1-}$ heart was markedly abnormal, with dilated and congested atria (Figure 2A, panels d-e). Following hematoxylin and eosin staining of the heart of E18.5 wild-type and Kidins $220^{-1-}$ littermates, we found that the ventricle walls were vacuolated and disorganized, when compared with wild-type tissue (Figure 2A, compare panels $c$ and $\mathrm{f}$ ). The dilation and congestion of the atria could be secondary to ventricle malfunctioning and increase in telediastolic ventricular 

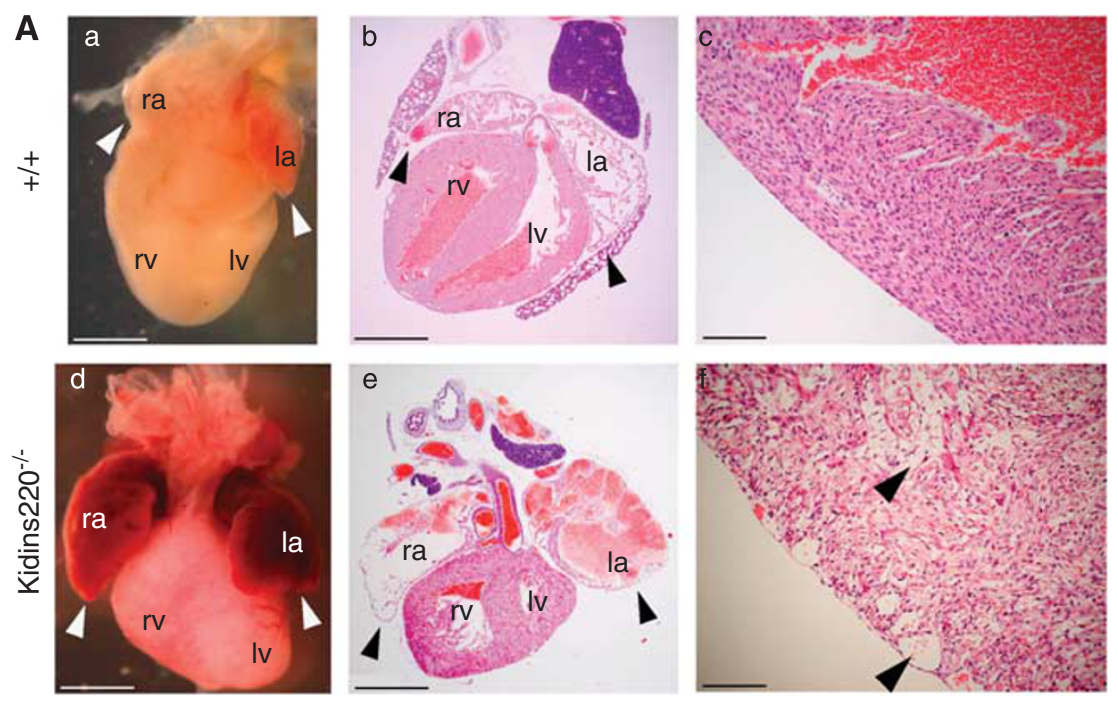

B
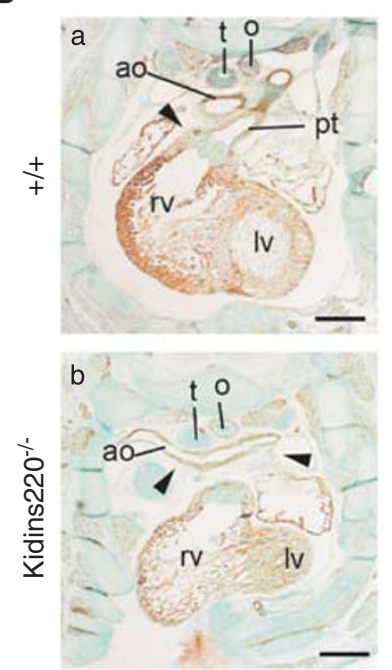

C

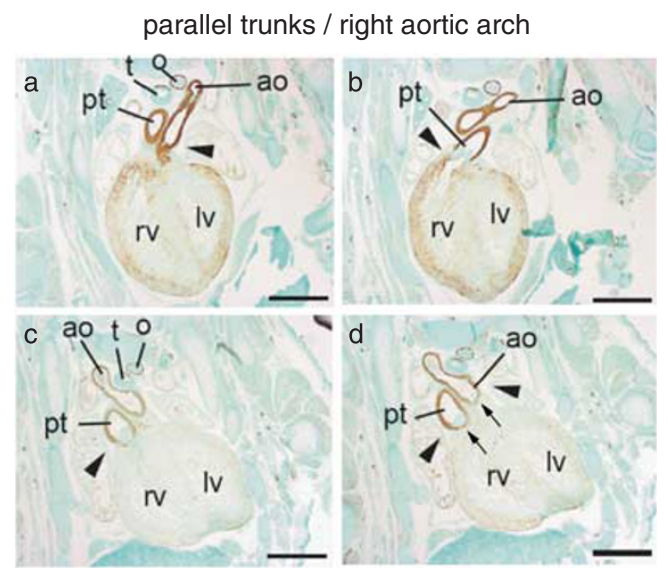

Figure 2 Heart defects in Kidins220 $0^{-1-}$ embryos. (A) Macroscopic view (a and d) and haematoxylin-eosin staining of sections (b, c and e, f) of E18.5 wild-type ( $+/+$ ) and Kidins220 ${ }^{-1-}$ hearts. Kidins220 ${ }^{-1-}$ hearts show congested and dilated atria (compare a and d, b and e, arrowheads). (c and f) Higher magnification of the right ventricle wall. In the Kidins $220^{-1-}$ ventricle, myocytes appear disorganized and loose, with enlarged interstitial spaces (f, arrowheads). Scale bars $=1 \mathrm{~mm}$ in a and $b$, and $d$ and $e$; $100 \mu \mathrm{m}$ in c and f. (B) E14.5 $\alpha$-smooth muscle actin staining reveals the presence of double aortic arch in Kidins220 ${ }^{-1-}$ hearts. The aortic arch splits to either side of the trachea and oesophagus ( $b$, arrowheads), while the aorta of wild-type heart runs on the left side of the oesophagus and trachea as expected (a, arrowhead). Scale bars, $250 \mu \mathrm{m}$. (C) E15.5 $\alpha$-smooth muscle actin staining evidenced right aortic arch and parallel arterial trunks, whereby the ascending aorta and pulmonary trunk are not spiralling (d, arrowheads). Scale bar, $250 \mu \mathrm{m}$. ao, aortic arch; la, left atrium; Iv, left ventricle; o, oesophagus; pt, pulmonary trunk; ra, right atrium; $\mathrm{r}$, right ventricle; t, trachea

pressure. Thus, heart failure could potentially explain the perinatal lethality of Kidins $220^{-1-}$ mice, as weak or defective blood pumping caused by these ventricle abnormalities would not allow mutant embryos to survive the stress of birth.

To further investigate the cardiac phenotype, E14.5-15.5 hearts from wild-type and Kidins $220^{-1-}$ embryos were stained for $\alpha$-smooth muscle actin, a marker of the myocardium and the smooth muscle layers of the pharyngeal arch arteries. A defect in outflow tract formation was present in Kidins220 ${ }^{-/-}$ mice. Although in wild-type sections a left-sided aortic arch could be observed as expected (Figure 2Ba), Kidins $220^{-1-}$ mice showed either a double-sided aortic arch whereby the right-sided 4th pharyngeal arch artery failed to regress as it should (Figure 2Bb), or a right-sided aortic arch, whereby the aortic arch developed from the right 4th pharyngeal arch artery, rather than the left, and was thus located on the right side of the oesophagus and trachea (Figure 2Cc and d, arrowheads). Additionally, both the pulmonary and aortic valves were observed on the same section of Kidins $220^{-1-}$ heart (Figure 2Cd, arrows), suggesting the presence of parallel arterial trunks (Figure 2Cc and d, arrowheads). As defects in heart development were found in mice lacking NT3, ${ }^{8}$ TrkC, $^{9}$ BDNF $^{6}$ and TrkB $^{7}$ our results suggest that Kidins220 has an important role in neurotrophin signaling during embryonic heart development.

Defects in sensory neurons development in Kidins $220^{-I-}$ embryos. A significant increase in apoptotic cells was found in both thoracic and lumbar DRGs in Kidins $220^{-1-}$ embryos. ${ }^{1}$ DRGs are composed of different pools of neurons, 
A
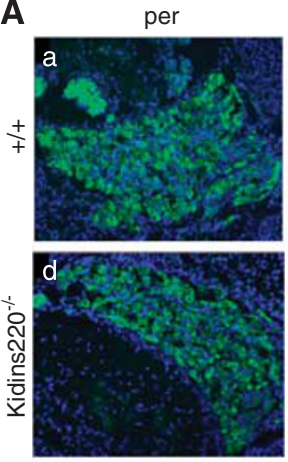

NFH
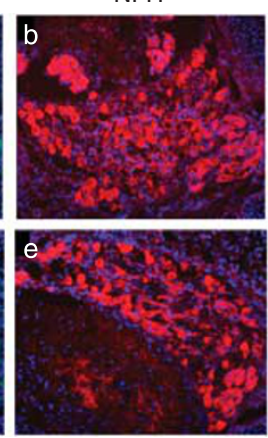

merge
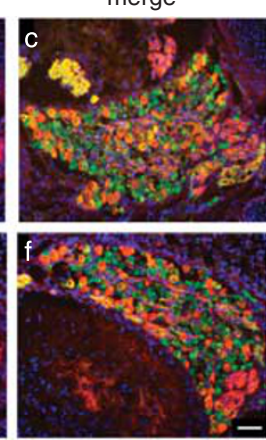

C
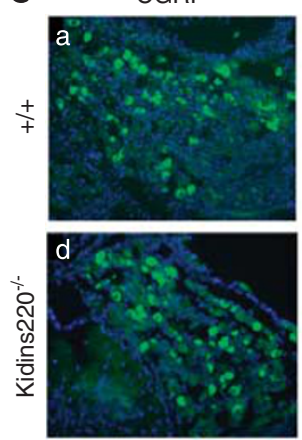

PV
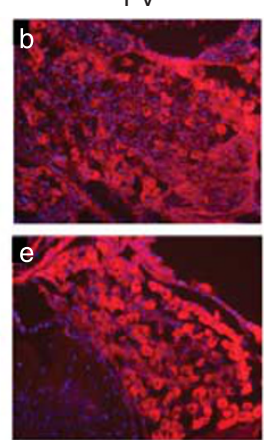

merge
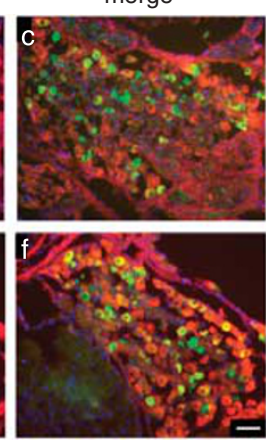

B
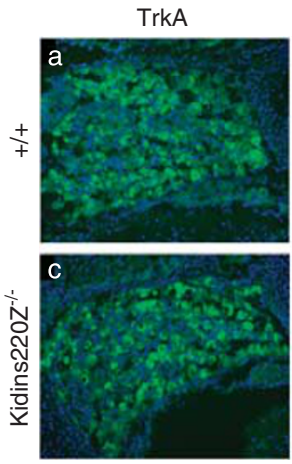

p75
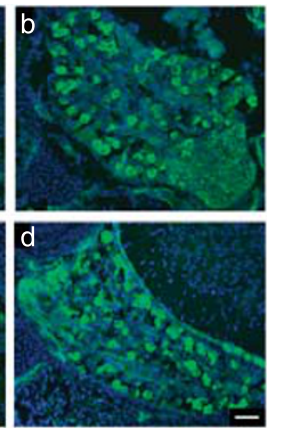

D

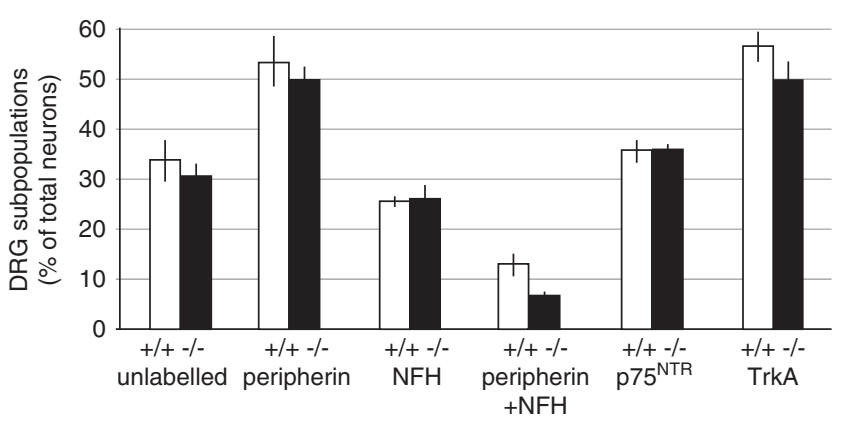

Figure 3 Immunohistochemical analysis of wild-type and Kidins220 ${ }^{-/}$DRGs. (A-C) L4-L6 DRGs from E18.5 wild-type (+/ + ) and Kidins220 ${ }^{-/-}$littermates were sectioned and stained with the indicated antibodies. Subpopulations of DRG neurons were identified based on the following markers: peripherin (per; unmyelinated and some small neurons), neurofilament (NFH; myelinated neurons), p75 ${ }^{\mathrm{NTR}}$ and parvalbumin (PV; proprioceptive and mechanoreceptive neurons), TrkA and calcitonin gene-related peptide (CGRP; mainly nociceptive and thermoreceptive neurons). (D) The relative proportion of distinct DRG subpopulations was unchanged in wild-type and Kidins220 ${ }^{-/-}$ E18.5 ganglia. $n=5$ embryos for each genotype; means \pm S.E.M. Scale bars, $100 \mu \mathrm{m}$

characterized by distinct neurotrophin requirement. ${ }^{27}$ As selective death of specific neuronal subpopulations were reported in mouse lines lacking NT or their receptors, ${ }^{27}$ we asked whether the lack of Kidins220 specifically affected one or more of these populations. To this end, we sectioned lumbar DRGs from wild-type and Kidins220 ${ }^{-1-}$ littermates at late stages of development (E18.5) and examined markers specific for different neuronal types, such as peripherin (per; unmyelinated and some small neurons), neurofilament (NFH; myelinated neurons), $\mathrm{p} 75^{\mathrm{NTR}}$ and parvalbumin (PV; proprioceptive and mechanoreceptive neurons), TrkA and calcitonin gene-related peptide (CGRP; mainly nociceptive and thermoreceptive neurons) (Figure 3). No significant differences were found between the relative abundance of these neurons in wild-type and mutant DRGs. In addition, despite the significant increase in the number of apoptotic cells in DRGs of Kidins $220^{-1-}$ mice, the cell area distribution of NFH- and peripherin-positive cell profiles was unchanged in the two genotypes (data not shown). This indicates that cell death in DRGs impacted equally on all the neuronal subtypes analysed (Figure 3). As none of the major DRG subpopulations displayed preferential cell loss in Kidins $220^{-1-}$ mice, we suggest that Kidins220 acts as a general survival factor during the early stages of sensory neuron development.

NT signaling is required for the development of peripheral sensory ganglia. ${ }^{27}$ Thus, we investigated whether Kidins220 ablation affects the survival of these neurons during development. To this end, we performed active caspase 3 staining on E14.5 brain sections, and revealed an increase in the number of apoptotic cells in the vestibular, glossopharyngeal and trigeminal ganglia (Figure 4), although the effect was statistically significant only for the first two ganglia. These data further confirm the importance of Kidins220 in mediating the survival of sensory neurons during embryonic development, which is likely to occur through neurotrophin receptor signaling. ${ }^{1}$

Role of Kidins220 in embryonic brain development. Kidins $220^{-1-}$ embryos display widespread cell death in the brain at late stages of development. ${ }^{1}$ To gain further insights into this phenomenon, we sought to characterise the time course of neuronal apoptosis in mutant embryos. To this end, we performed active caspase 3 staining on brain sections from embryos at various developmental stages. We did not find any cell death at E13.5 in mutant tissues (Figure 5), suggesting that Kidins 220 is not essential in the early stages of brain morphogenesis. However, a clear pattern of neuronal apoptosis affecting the neuroepithelium, retina and thalamus was clearly visible already at E15.5 (Figure 6), and progressively worsened during the last stages of embryonic development, leading to the massive cell death observed at E18.5. ${ }^{1}$ Taken altogether, these data indicate that the physiological function of Kidins220 in promoting neuronal survival starts at mid-gestation and is maintained until birth. 

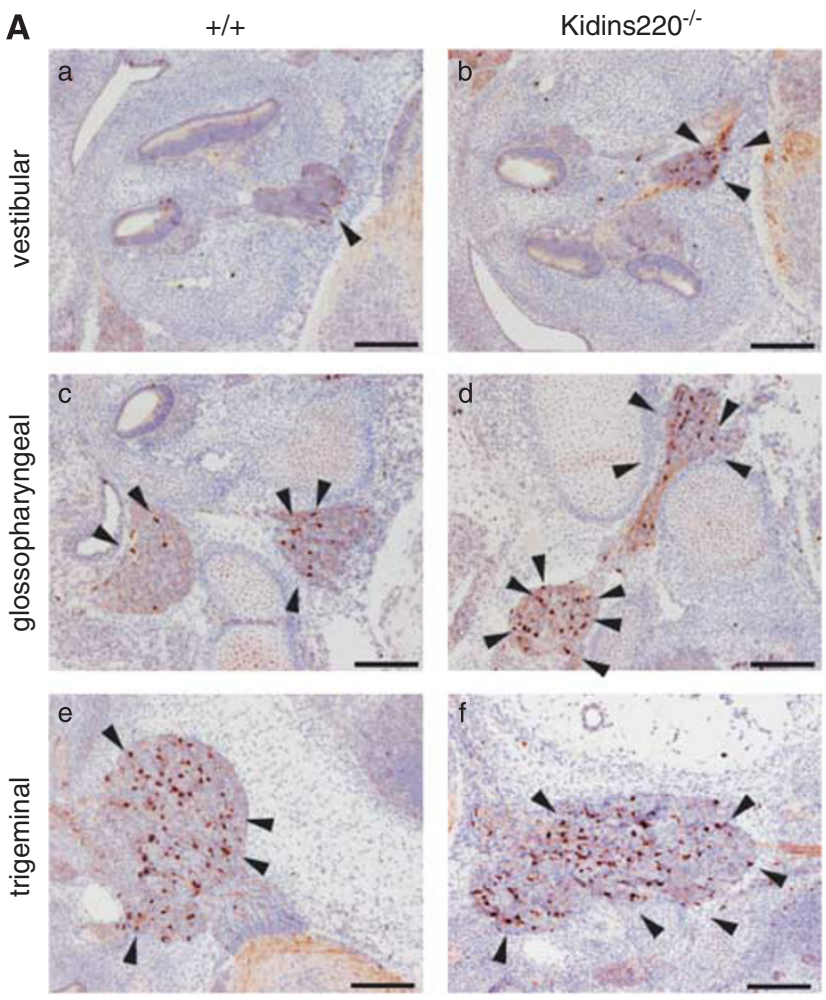

B

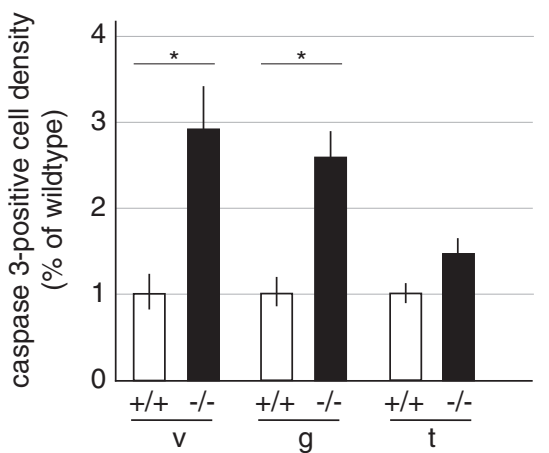

Figure 4 Kidins $220^{-1-}$ mice display increased apoptosis in cranial sensory ganglia. (A) Brain sagittal sections from E14.5 wild-type $(+/+)$ and Kidins220 $20^{-1}$ littermates were immunostained for active caspase 3 . Increased apoptosis could be observed in the vestibular ( $a$ and b), glossopharyngeal ( $c$ and $d$ ) as well as in the trigeminal (e and f) ganglia. Scale bars, $100 \mu \mathrm{m}$. (B) The number of caspasepositive cells were counted and normalized to the area of the ganglia visible in each section. Data are expressed as means \pm S.E.M. A statistically significant increase in the percentage of apoptotic cells was observed in the vestibular (v) and glossopharyngeal $(\mathrm{g})$ ganglia of Kidins $22 \mathrm{O}^{-1-}$ mice, compared with wild-type $\left({ }^{\star} P<0.05\right.$, Student's unpaired $t$-test, $10-20$ sections/embryo, $n=3$ embryos for each genotype). An increase in the percentage of apoptotic cells was also observed in the trigeminal ( $t$ ) ganglia, although it did not reach statistical significance

Role of Kidins220 in postnatal brain development. Our data and work from others ${ }^{26}$ have shown that Kidins 220 expression is high during embryogenesis, and progressively declines during the first two postnatal weeks. Despite the low expression in adult brain, a $30-40 \%$ reduction of its protein levels was, however, sufficient to affect cortical neuron development and synaptic plasticity. ${ }^{26}$ To assess the effects of the complete ablation of Kidins 220 in postnatal
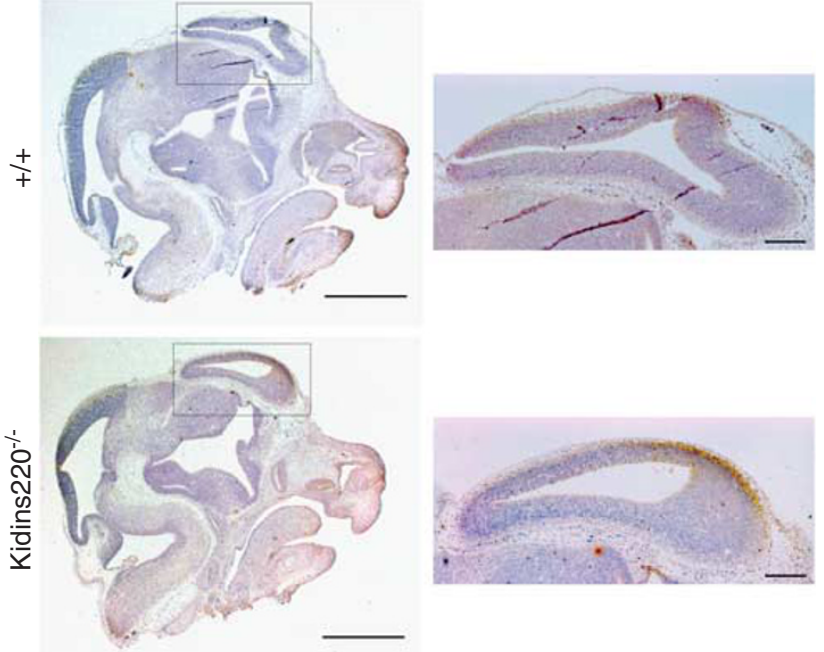

Figure 5 Absence of cell death in Kidins220 ${ }^{-1-} \mathrm{E} 13.5$ brains. Brains from wildtype or Kidins220 ${ }^{-1-}$ E13.5 embryos were sectioned and stained with anti-active caspase 3 antibodies. No immunoreactive cells were present at this developmental stage in either genotypes, as exemplified by the high magnification images of the forebrain region (right panels). Scale bars, $1 \mathrm{~mm}$ (left panels) and $200 \mu \mathrm{m}$ (right panels). Staining was performed on $n>3$ embryos per genotype from at least two different litters

development, we generated a nervous system-specific knockout line (Kidins $220^{\Delta N}$ ) by crossing Kidins $220^{\text {lox/lox }}$ mice to animals carrying the Cre recombinase under the control of the Nestin promoter (Nestin-Cre ${ }^{+/-}$). ${ }^{28}$ The correct pattern of recombination was confirmed by mating Nestin$\mathrm{Cre}^{+/-}$mice to Rosa26-LacZ reporter mice ${ }^{29}$ and by western blot analysis (data not shown). Although the distribution of genotypes in embryos was fairly close to the expected Mendelian ratio, genotyping of P0-P2 pups revealed a percentage of Kidins $220^{\Delta \mathrm{N}}$ animals lower than expected, indicating that some of the Kidins $220^{\Delta \mathrm{N}}$ pups died soon after birth. The Kidins $220^{\Delta \mathrm{N}}$ mice that survived appeared similar in size to their wild-type littermates, and showed normal movements of their fore- and hindlimbs. However, they showed early postnatal lethality, and no animals survived beyond P2. Kidins $220^{\Delta N}$ hearts appeared normal with no dilation of the atria (Figure 7A), and no visible defects in the ventricular chamber walls (Figure 7B), strongly suggesting that a cardiac phenotype is not the cause of the perinatal death of these mutant animals. Post-mortem analysis of mutant pups showed no milk in the stomach, thus indicating that their death might be due to a neurological phenotype impairing suckling.

Brains from Kidins $220^{\Delta \mathrm{N}}$ mice appeared smaller than their Kidins $220^{\text {lox/lox }}$ littermates, similar to the Kidins $220^{-1-}$ brains. ${ }^{1}$ In order to assess if the nervous system-specific ablation of Kidins 220 caused a cell death phenotype similar to the full knockout, we stained coronal brain sections of E16.5

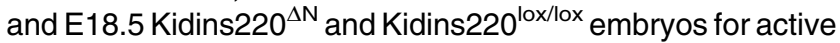
caspase 3 (Figure 7C and D). Surprisingly, we found a reduced cell death in Kidins $220^{\Delta \mathrm{N}}$ brains. Although an increase in apoptosis was observed in the cingulate cortex compared with wild-type samples (E16.5; Figure 7D, compare $a$ and $b$ ), the number of dying cells was lower than in 
A

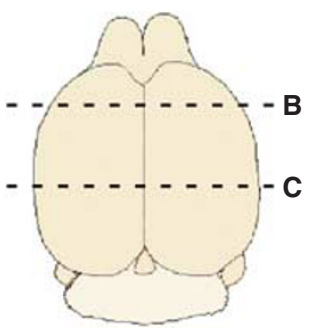

B a
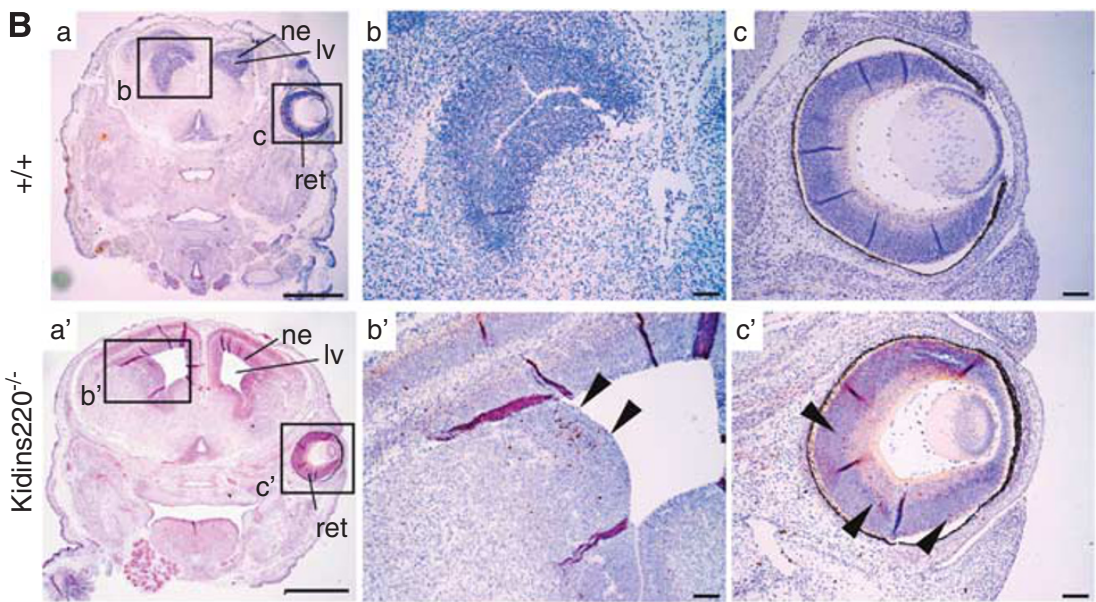

C
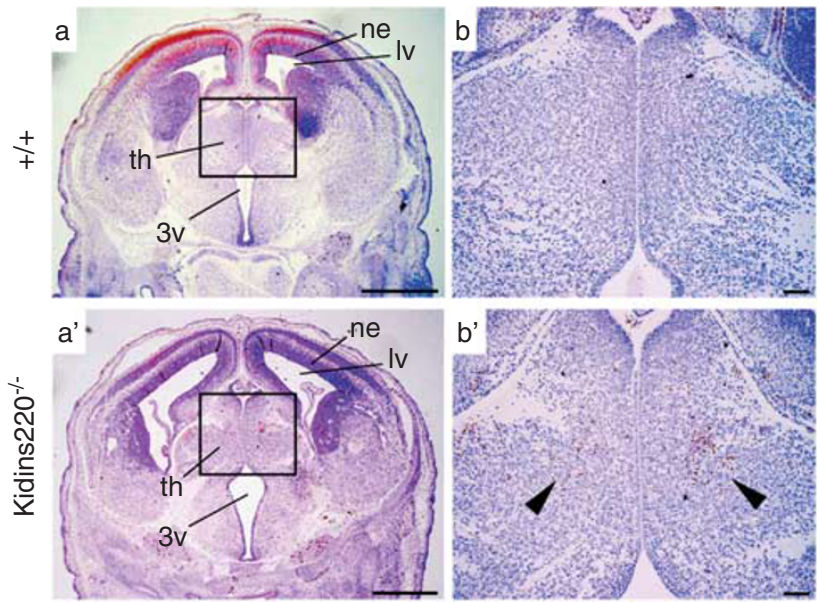

Figure 6 Distinct neuronal subpopulations die in Kidins220 ${ }^{-1}$ E15.5 brains. Brains from wild-type or Kidins220 ${ }^{-1-}$ E15.5 embryos were sectioned and stained with antiactive caspase 3 antibodies. Staining was performed on $n>3$ embryos per genotype from at least two different litters. (A) Dashed lines indicate the approximate planes of sections for the images shown in (B) and (C). (B) Caspase 3-positive cell clusters are visible in the neuroepithelium, adjacent to the lateral ventricle (a' and arrowheads in b), as well as in the retina ( $c^{\prime}$, arrowheads) of Kidins220 ${ }^{-1-}$ samples, which are absent in the wild-type (a-c). (C) Distinct pools of caspase 3-positive cells are present in the developing thalamus (a'and arrowheads in b). Panels $b$ and c, and b' and c' are higher magnification images of the boxed regions in panels a and a'. Scale bars in panels a and $a^{\prime}=1 \mathrm{~mm}$; in panels $b$ and b', and $c$ and $c^{\prime}=100 \mu \mathrm{m}$. $3 \mathrm{v}$, third ventricle; Iv, lateral ventricle; ne, neuroepithelium; ret, retina; th, thalamus

Kidins $220^{-1-}$ samples. ${ }^{1}$ In more caudal sections, increased apoptosis in the VPL/VPM nuclei of the thalamus was observed in Kidins220 $0^{\Delta \mathrm{N}}$ brains (Figure 7D, compare a and $b$, arrowheads) compared with wild-type sections. However, no apparent cell death was observed in the reuniens nucleus surrounding the third ventricle (Figures $7 \mathrm{C}$ and Dc, d, arrows), in the neuroepithelium adjacent to the lateral ventricle and in the hippocampus (Figure $7 \mathrm{Ca}$ and $\mathrm{b}$ ).

These findings suggest that the ablation of Kidins220 in neurons and glia is not the only responsible for the death phenotype observed in the brain of Kidins220 ${ }^{-1-}$ embryos, and that the absence of Kidins220 from other cell types is likely to have a prominent role in this phenomenon. As haemorrhaging is commonly observed in Kidins $220^{-1-}$ brains, ${ }^{1}$ we then tested whether a similar phenotype was present in the Kidins $220^{\Delta \mathrm{N}}$ mice. For this purpose, we performed isolectin-B4 staining on E16.5 coronal sections, which revealed a largely normal vascular network, with no evidence of glomeruloid terminal structures in either the subventricular zone or thalamic region (Figure 7E). 

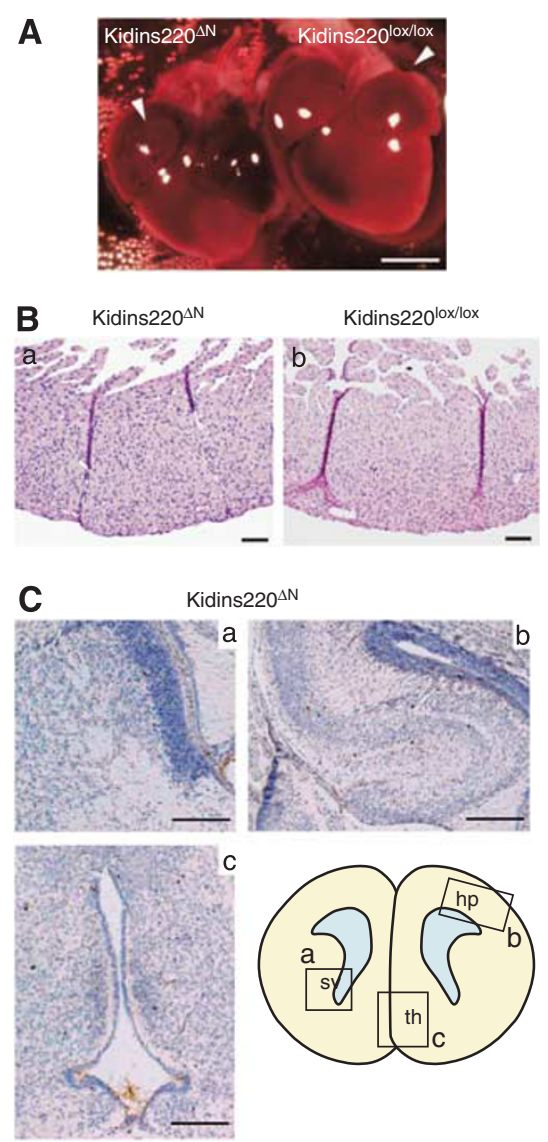

D
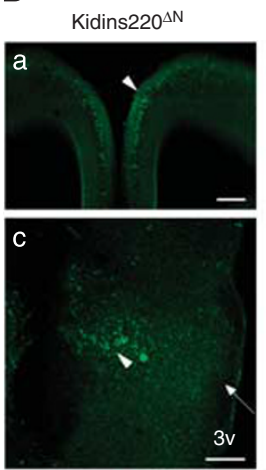

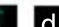
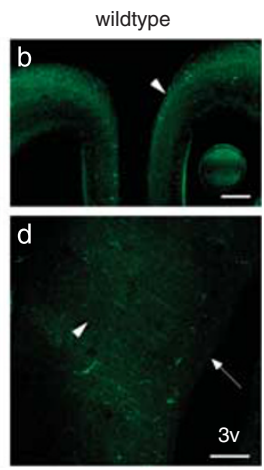

E Kidins $220^{\Delta \mathrm{N}}$

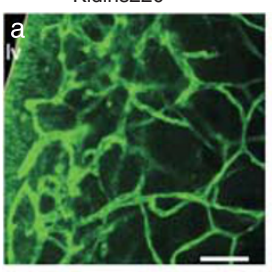

Kidins220 lox/lox
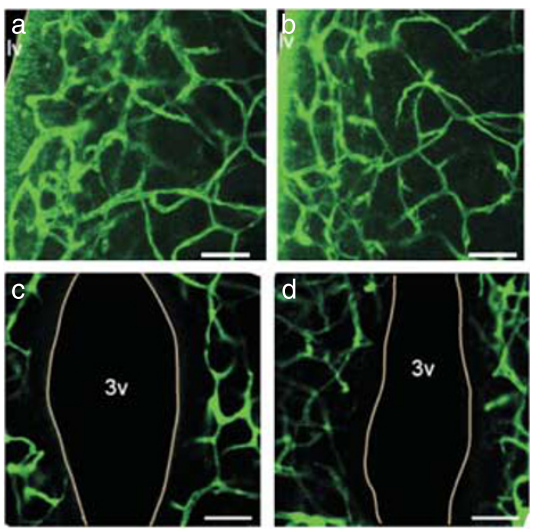

Figure 7 Histological analysis of Kidins $220^{\Delta N}$ animals. (A and B) Macroscopic view and haematoxylin-eosin stained sections of PO Kidins220 ${ }^{\Delta N}$ and Kidins $220^{\text {lox/lox }}$ hearts. No dilation of atria is seen in Kidins $220^{\Delta \mathrm{N}}$ samples $(A)$, and their ventricular wall appears largely normal (B). Scale bars, $500 \mu \mathrm{m}$ in $(A), 20 \mu \mathrm{m}$ in $(B)$. (C) Brains from Kidins $220^{\Delta \mathrm{N}}$ E18.5 embryos were sectioned and stained with anti-active caspase 3 antibodies. No immunoreactivity was observed in Kidins220 ${ }^{\Delta \mathrm{N}}$ samples in the subventricular zone (sv, a), hippocampus (hp, b), and reuniens nucleus of the thalamus (th, c) at this developmental stage. (D) Active caspase 3 staining on E16.5 wild-type and Kidins $220^{\Delta \mathrm{N}}$ brain sections revealed increased apoptosis in the cingulate cortex of Kidins $220^{\Delta \mathrm{N}}$ compared with wild-type (compare a, arrowhead, to b), and in the VPL/VPM of the thalamus (compare $c$ and d arrowheads), while no cell death was present at the reuniens nucleus (compare $c$ and $d$, arrows). Scale bars, $200 \mu$ m. Absence of death in Kidins220 $20^{\text {lox/lox }}$ was also confirmed (data not shown). (E) E16.5 Kidins220 lox/lox and Kidins $220^{\Delta \mathrm{N}}$ brain sections were stained with isolectin-B4. The outer limits of the brain have been outlined in grey according to the phase image. Both the neuroepithelium surrounding the lateral ventricles (a and $b$ ) and the reuniens nucleus surrounding the third ventricle ( $c$ and d) show similar vascular pattern in Kidins $220^{\Delta \mathrm{N}}$ brains compared with Kidins $220^{\text {lox/lox }}$. No vascular glomeruloid plexus is visible. Iv, lateral ventricle; $3 \mathrm{v}$, third ventricle. Scale bars, $50 \mu \mathrm{m}$

Altogether, these results indicate that the massive apoptosis characterising full knockout brains is caused by a combination of neuronal and non-neuronal (mostly vascular) defects.

\section{Discussion}

In this work, we have conducted a detailed analysis of heart, CNS and PNS development in a novel Kidins $220^{-1-}$ mouse line. ${ }^{1}$ In the heterozygous mice, the protein levels of Kidins220 were comparable to wild-type animals, in all brain regions and at all ages analysed. This suggests that the expression of Kidins220 is tightly regulated at both translational and posttranslational levels, which is not unexpected given the multiple interactions and pathways engaged by this protein. A small reduction in Kidins220 protein levels, or perhaps an imbalance between different isoforms, might shift the precise equilibrium in the signals originating from different receptors. The finding that even a partial reduction of Kidins220 levels is sufficient to cause significant impairment in cortical development and synaptic plasticity further supports this hypothesis. ${ }^{26}$

Role of Kidins220 in heart development. Kidins $220^{-1-}$ mice display severe morphological heart defects, which include enlarged atria and thinning of the ventricular myocardial wall. In addition, Kidins $220^{-1-}$ hearts showed deficiencies in the cardiac outflow tract such as double aortic arch, right-sided aortic arch and parallel trunks. BDNF ${ }^{-1-}$ and $\mathrm{TrkB}^{-1-}$ animals suffer early postnatal death due to the death of cardiac endothelial cells and defective development of heart vasculature, which cause haemorrhages and impaired heart contractility. ${ }^{6,7}$ Deficiencies in heart development were also found in $\mathrm{NT}^{-1-}$ and $\mathrm{TrkC}^{-1-}$ mice, which display enlarged globular hearts, outflow tract defects (overriding aorta), valve malformations and atrial and ventricular septal defects, which can, for the most part, be related to neural crest abnormalities. ${ }^{8,9}$ Although the exact 
function of neurotrophins in neural crest migration and differentiation is still unclear, cardiac outflow tract defects such as those observed in Kidins $220^{-1-}$ hearts are typical of mice with defective cardiac neural crest cells, ${ }^{30}$ suggesting a major role of Kidins220 in processes involving this important cell type. The Eph/ephrin pathway is also required for normal cardiovascular development, but the heart defects described in Eph- and ephrin-deficient lines are much more severe than those found in the NT-, Trk- or Kidins220-deficient animals. ${ }^{31}$ For this reason, we deem it unlikely that the malformations in the heart of Kidins $220^{-1-}$ mice are related to defects in Eph/ ephrin signaling.

Role of Kidins220 in sensory ganglia development. The phenotypes of NT- and Trk-deficient mice are much more evident in the PNS than in the CNS. This is probably due to the fact that, while in the brain the numerous trophic factors can compensate for each other, sensory neurons usually show a specific dependence on one or more neurotrophin(s). ${ }^{3}$ As Kidins220 interacts with all Trks and with p75 ${ }^{\text {NTR }}, 18,19$ its ablation is expected to cause a range of phenotypes recapitulating the role of all these receptors. In accordance with this hypothesis, we found widespread apoptosis in all types of DRG neurons, similar to the phenotype found in mice lacking $\mathrm{p} 75^{\mathrm{NTR}}$, another pan-Trk interactor. ${ }^{3}$ Moreover, the trigeminal, glossopharyngeal and vestibular ganglia also show increased apoptosis, as reported in multiple NT knockout mice. ${ }^{9,32,33}$ Altogether, our results indicate that Kidins220 is a fundamental mediator of the NT pathways in the development of the PNS.

Role of Kidins220 in postnatal brain development and plasticity. The early postnatal death of Kidins $220^{\Delta \mathrm{N}}$ mice indicates that the physiological functions of Kidins220 extend beyond embryonic development. At the same time, however, it precludes any analysis of the role of Kidins 220 in the adult brain. The generation of various Kidins220-deficient lines will allow us to bypass the problem of early lethality, and permit an in-depth analysis of the functions of this protein during postnatal development and in adulthood. It will be of interest to verify whether the neuronal-specific deletion of Kidins220 will cause neurodegeneration, and whether adult mice lacking Kidins220 will show changes in the electrophysiological properties of their mature neuronal networks due to an imbalance of excitatory and inhibitory inputs. As these alterations have been found in many neurological disorders including epilepsy, autism and schizophrenia, our functional in vivo analysis of Kidins220-deficient animals might be important to further our understanding of the physiopathology of these diseases and open the possibility of using Kidins220 as a biomarker in brain and spinal cord pathologies.

\section{Materials and Methods \\ Materials. All biochemical reagents were from Sigma (Sigma, Milan, Italy),} unless otherwise specified.

Antibodies. Fluorescently-conjugated antibodies for immunofluorescence were from Molecular Probes (Invitrogen, Carlsbad, CA, USA). Fluorescently-conjugated antibodies for western blot analysis and immunocytochemistry were ECL Plex goat $\alpha$-rabbit IgG-Cy5 (PA45012, GE Healthcare, Milan, Italy), goat $\alpha$-chicken IgGDyLight488 and donkey $\alpha$-mouse IgG-Cy3 (103-485-155 and 715-166-150, Jackson ImmunoResearch, Suffolk, UK). Monoclonal and polyclonal Kidins220 antibodies were previously described. ${ }^{24,25}$ The following primary antibodies were used: polyclonal anti-neuronal class III $\beta$-tubulin (T2200, Sigma), polyclonal antiactive caspase 3 (AF835, R\&D Systems, Minneapolis, MN, USA), monoclonal antineurofilament $H$ (N0142, Sigma), polyclonal anti-p $75^{\text {NTR }}$ (G3231, Promega, Madison, WI, USA), polyclonal anti-TrkA (Advanced Targeting Systems, San Diego, CA, USA), monoclonal anti-PV (235, Swant, Bellinzona, Switzerland), polyclonal anti-CGRP (1134, Enzo Life Sciences, Exeter, UK), polyclonal anti-per (AB1530, Millipore, Billerica, MA, USA), monoclonal anti- $\alpha$-smooth muscle actin (A2547, Sigma Aldrich, Milan, Italy).

Gene targeting. The generation of the Kidins $220^{+/-}$is described. ${ }^{1}$ Kidins $220^{\Delta \mathrm{N}}$ line was generated by crossing Kidins $220^{\text {lox/lox }}$ mice with Nestin-Cre animals. ${ }^{28}$ All embryos used in this study were obtained from crosses of Kidins220 ${ }^{+/-}$mice on the C57BL/6 background. Mice were mated overnight and separated in the morning. Embryos were timed from the detection of a vaginal plug, which was considered day 0.5. Experiments performed on animals and embryos in the UK were under license from the UK Home Office (Animals Scientific Procedures Act 1986), approved by the Cancer Research UK Ethical Committee, whereas those done in Italy were conducted in accordance with the European Community Council Directive dated November $24,1986(86 / 609 / \mathrm{EEC})$ and approved by the Italian Ministry of Health.

Biochemical techniques. Mouse brain tissues were extracted in RIPA buffer ( $50 \mathrm{mM}$ Tris-HCl pH 7.4, $150 \mathrm{mM} \mathrm{NaCl}, 2 \mathrm{mM}$ EDTA, NP40 1\%, SDS 0.1\%) plus protease inhibitors (complete EDTA-free protease inhibitors, Roche Diagnostic, Milan, Italy) using a teflon dounce homogenizer (Wheaton, Millville, NJ, USA). After centrifugation at $16000 \mathrm{~g}$ for $15 \mathrm{~min}$ at $4{ }^{\circ} \mathrm{C}$, protein concentration was quantified using the Bradford Protein Assay (BioRad, Hercules, CA, USA). SDS-PAGE and western blotting were performed by using precast $4-12 \%$ NuPAGE Novex Bis-Tris Gels (Invitrogen). After incubation with primary antibodies, membranes were incubated with fluorescently-conjugated secondary antibodies and revealed by a Typhoon TRIO + Variable Mode Imager (GE Healthcare). Immunoreactive bands were quantified by using the ImageQuant TL software (GE Healthcare).

Immunohistochemistry. Paraffin embedding, sectioning $(4 \mu \mathrm{m})$ and haematoxylin/eosin staining were performed according to standard procedures. For immunohistochemistry, sections were microwaved in citrate buffer $(10 \mathrm{mM}$ trisodium citrate $\mathrm{pH} 6,4.5 \mathrm{mM} \mathrm{HCl}$ ) for $10 \mathrm{~min}$ for antigen retrieval. After blocking endogenous peroxidase with normal serum, sections were incubated with rabbit anti-active caspase 3 primary antibody at $1 / 800$ for $1 \mathrm{~h}$ at room temperature and subsequently incubated with the appropriate biotin-conjugated goat anti-rabbit secondary antibody, tertiary $A B C$ elite reagent (Vector Laboratories, Peterborough, UK) and developed with DAB (Biogenix, Montreal, QC, Canada). Tissue sections were analyzed using a Nikon Eclipse E1000 microscope equipped with a Nikon (Nikon Instruments S.p.a., Firenze, Italy) digital camera DXM1200F, and the following Nikon Plan-Apochromat objectives: $4 \times 0.2$ NA DIC, $10 \times 0.45$ NA DIC, $20 \times 0.75$ NA DIC, Nikon $40 \times 0.95$ NA DIC.

Vibratome sectioning and staining. Brains were fixed in 4\% PFA in PBS overnight at $4{ }^{\circ} \mathrm{C}$, washed and mounted in $3.5 \%$ low melt agarose in PBS. $200 \mu \mathrm{m}$ sections were made using a vibratome (Leica VT1000S, Milton Keynes, UK). Floating sections were blocked in $1 \% \mathrm{BSA}, 0.5 \%$ Triton $\times 100$ in PBS for $2 \mathrm{~h}$. After conditioning into Pblec (PBS pH 6.8, $1 \mathrm{mM} \mathrm{CaCl}, 1 \mathrm{mM} \mathrm{MgCl}_{2}, 0.1 \mathrm{mM} \mathrm{MnCl}_{2}, 1 \%$ Triton $\times 100$ ), sections were incubated overnight with 1:1000 AlexaFluor-488 conjugated Isolectin B4 from $\mathrm{G}$. simplicifolia (I21411, Invitrogen) at $4{ }^{\circ} \mathrm{C}$, washed in PBS, incubated with primary and secondary antibodies in $0.5 \%$ BSA, $0.25 \%$ Triton $\times 100$ in PBS and mounted in Mowiol 4-88 (Dako, Milan, Italy).

DRG analysis. Lumbar spinal regions of E18.5. embryos were fixed in 4\% PFA and put through a sucrose gradient to $30 \%$ sucrose overnight at $4{ }^{\circ} \mathrm{C}$. Samples were embedded in OCT, $10 \mu \mathrm{m}$ sections were cut on a cryostat and immunostained at room temperature following permeabilisation with $0.3 \%$ Triton $\times 100$ and blocking with pre-immune donkey serum. Slides were mounted using a Vectashield mounting media with DAPI and viewed on a Zeiss Axiophot 2.

Lumbar DRG from each embryo were analysed separately. Captured images were viewed in Adobe Photoshop CS4. A minimum of 500 cells in three DRG 
sections were analysed for each animal and the number of cell profiles positive for each marker was recorded. Of those profiles showing nuclei, the areas and perimeters of positive cells for each marker were recorded using a 21 -inch LCD digitalising tablet.

\section{Conflict of Interest}

The authors declare no conflict of interest.

Acknowledgements. We thank $\mathrm{J}$ Storm-Mathisen for help with the anatomical analysis, L Mair and S Stewart for DRG experiments, A Behrens for the Nestin-Cre mice, CL Thomas for critical reading of the manuscript and members of the Molecular Neuropathobiology laboratory for helpful discussion. This study was supported by research grants from: Cancer Research UK (FC, AY, BS-D and GS); the Italian Institute of Technology (FC and FB); the Italian Ministry of University and Research (FB); the Compagnia di San Paolo, Torino (FB); Telethon-Italy (Grant GGP09134 to FB); BBSRC (MAQ and MK); the British Heart Foundation (Programme Grant RG/07/07 to DH).

1. Cesca F, Yabe A, Spencer-Dene B, Scholz-Starke J, Medrihan L, Maden $\mathrm{CH}$ et al Kidins220/ARMS Mediates the Integration of the Neurotrophin and VEGF Pathways in the Vascular and Nervous Systems. Cell Death Diff 2011; e-pub ahead of print 3 November 2011; doi:10.1038/cdd.2011.141.

2. Cohen S, Levi-Montalcini R, Hamburger V. A Nerve Growth-Stimulating Factor Isolated from Sarcom as 37 and 180. Proc Natl Acad Sci USA 1954; 40: 1014-1018.

3. Huang EJ, Reichardt LF. Neurotrophins: roles in neuronal development and function. Annu Rev Neurosci 2001; 24: 677-736.

4. Enokido Y, Wyatt S, Davies AM. Developmental changes in the response of trigeminal neurons to neurotrophins: influence of birthdate and the ganglion environment Development 1999; 126: 4365-4373.

5. Molliver DC, Wright DE, Leitner ML, Parsadanian AS, Doster K, Wen D et al. IB4-binding DRG neurons switch from NGF to GDNF dependence in early postnatal life. Neuron 1997; 19: 849-861.

6. Donovan MJ, Lin MI, Wiegn P, Ringstedt T, Kraemer R, Hahn R et al. Brain derived neurotrophic factor is an endothelial cell survival factor required for intramyocardial vessel stabilization. Development 2000; 127: 4531-4540.

7. Wagner N, Wagner KD, Theres H, Englert C, Schedl A, Scholz H. Coronary vessel development requires activation of the TrkB neurotrophin receptor by the Wilms' tumor transcription factor Wt1. Genes Dev 2005; 19: 2631-2642.

8. Donovan MJ, Hahn R, Tessarollo L, Hempstead BL. Identification of an essential nonneuronal function of neurotrophin 3 in mammalian cardiac development. Nat Genet 1996; 14: 210-213.

9. Tessarollo L, Tsoulfas P, Donovan MJ, Palko ME, Blair-Flynn J, Hempstead BL et al. Targeted deletion of all isoforms of the trkC gene suggests the use of alternate receptors by its ligand neurotrophin-3 in neuronal development and implicates trkC in normal cardiogenesis. Proc Natl Acad Sci USA 1997; 94: 14776-14781.

10. Youn YH, Feng J, Tessarollo L, Ito K, Sieber-Blum M. Neural crest stem cell and cardiac endothelium defects in the TrkC null mouse. Mol Cell Neurosci 2003; 24: 160-170.

11. von Schack D, Casademunt E, Schweigreiter R, Meyer M, Bibel M, Dechant G. Complete ablation of the neurotrophin receptor p75NTR causes defects both in the nervous and the vascular system. Nat Neurosci 2001; 4: 977-978.

12. Bronfman FC, Fainzilber M. Multi-tasking by the $p 75$ neurotrophin receptor: sortilin things out? EMBO Rep 2004; 5: 867-871.
13. Chao MV. Increasing the specificity of neurotrophic factors. Proc Natl Acad Sci USA 2010; 107: 13565-13566.

14. Dalva MB, Takasu MA, Lin MZ, Shamah SM, Hu L, Gale NW et al. EphB receptors interact with NMDA receptors and regulate excitatory synapse formation. Cell 2000; 103: 945-956.

15. Jin Y, Garner CC. Molecular mechanisms of presynaptic differentiation. Annu Rev Cell Dev Biol 2008; 24: 237-262.

16. Haucke V, Neher E, Sigrist SJ. Protein scaffolds in the coupling of synaptic exocytosis and endocytosis. Nat Rev Neurosci 2011; 12: 127-138.

17. Kim E, Sheng M. PDZ domain proteins of synapses. Nat Rev Neurosci 2004; 5: 771-781.

18. Kong H, Boulter J, Weber JL, Lai C, Chao MV. An evolutionarily conserved transmembrane protein that is a novel downstream target of neurotrophin and ephrin receptors. J Neurosci 2001; 21: 176-185

19. Arevalo JC, Yano H, Teng KK, Chao MV. A unique pathway for sustained neurotrophin signaling through an ankyrin-rich membrane-spanning protein. EMBO J 2004; 23: 2358-2368.

20. Chang MS, Arevalo JC, Chao MV. Ternary complex with Trk, p75, and an ankyrin-rich membrane spanning protein. J Neurosci Res 2004; 78: 186-192.

21. Luo S, Chen Y, Lai KO, Arevalo JC, Froehner SC, Adams ME et al. \{alpha\}-Syntrophin regulates ARMS localization at the neuromuscular junction and enhances EphA4 signaling in an ARMS-dependent manner. J Cell Biol 2005; 169: 813-824.

22. Arevalo JC, Wu SH, Takahashi T, Zhang H, Yu T, Yano H et al. The ARMS/Kidins220 scaffold protein modulates synaptic transmission. Mol Cell Neurosci 2010; 45: 92-100.

23. Lopez-Menendez C, Gascon S, Sobrado M, Vidaurre OG, Higuero AM, Rodriguez-Pena A et al. Kidins220/ARMS downregulation by excitotoxic activation of NMDARs reveals its involvement in neuronal survival and death pathways. J Cell Sci 2009; 122: 3554-3565.

24. Neubrand VE, Thomas C, Schmidt S, Debant A, Schiavo G. Kidins220/ARMS regulates Rac1-dependent neurite outgrowth by direct interaction with the RhoGEF Trio. J Cell Sci 2010; 123: 2111-2123.

25. Bracale A, Cesca F, Neubrand VE, Newsome TP, Way M, Schiavo G. Kidins220/ARMS is transported by a kinesin-1-based mechanism likely to be involved in neuronal differentiation. Mol Biol Cell 2007; 18: 142-152.

26. Wu SH, Arevalo JC, Sarti F, Tessarollo L, Gan WB, Chao MV. Ankyrin Repeat-rich Membrane Spanning/Kidins220 protein regulates dendritic branching and spine stability in vivo. Dev Neurobiol 2009; 69: 547-557.

27. Bibel M, Barde YA. Neurotrophins: key regulators of cell fate and cell shape in the vertebrate nervous system. Genes Dev 2000; 14: 2919-2937.

28. Tronche F, Kellendonk C, Kretz O, Gass P, Anlag K, Orban PC et al. Disruption of the glucocorticoid receptor gene in the nervous system results in reduced anxiety. Nat Genet 1999; 23: 99-103.

29. Soriano P. Generalized lacZ expression with the ROSA26 Cre reporter strain. Nat Genet 1999; 21: 70-71.

30. Conway SJ, Kruzynska-Frejtag A, Kneer PL, Machnicki M, Koushik SV. What cardiovascular defect does my prenatal mouse mutant have, and why? Genesis 2003; 35: 1-21.

31. Adams RH, Klein R. Eph receptors and ephrin ligands. essential mediators of vascular development. Trends Cardiovasc Med 2000; 10: 183-188.

32. Ernfors $P$, Lee KF, Jaenisch R. Mice lacking brain-derived neurotrophic factor develop with sensory deficits. Nature 1994; 368: 147-150.

33. Ernfors $P$, Lee KF, Kucera J, Jaenisch R. Lack of neurotrophin-3 leads to deficiencies in the peripheral nervous system and loss of limb proprioceptive afferents. Cell 1994; 77 : 503-512.

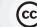

(c)

Cell Death and Disease is an open-access journal published by Nature Publishing Group. This work is licensed under the Creative Commons Attribution-Noncommercial-No Derivative Works 3.0 Unported License. To view a copy of this license, visit http://creativecommons.org/licenses/by-nc-nd/3.0/ 